\title{
Evaluación de la eficacia de la formación en la Administración Pública: la transferencia al puesto
}

\section{Evaluation of the effectiveness of training in the Spanish Public Administration: transfer to the job}

\author{
Aitana González-Ortiz de Zárate \\ Universidad Complutense de Madrid \\ aitana.gonzalez@ucm.es \\ Miguel Aurelio Alonso García \\ Universidad Complutense de Madrid \\ malonsog@ucm.es \\ Francisca Berrocal Berrocal
Universidad Complutense de Madrid
fberrocal@psi.ucm.es
}

\section{RESUMEN}

El artículo recoge los resultados de la evaluación de la transferencia de la formación en la Administración General del Estado con el fin de conocer los factores que en mayor medida influyen en que se produzca.

Se analizaron las respuestas de 1.475 participantes en 69 cursos desarrollados por el INAP (Instituto Nacional de la Administración Pública) durante el primer semestre de 2016. Se evaluó la transferencia directa con el Cuestionario de Eficacia de la Formación (CdE; Pineda, Quesada y Ciraso 2011) y la indirecta con el cuestionario de Factores de Eficacia de la Transferencia (FET; Pineda, Quesada y Ciraso, 2013).

Los resultados muestran que los factores de los participantes, del lugar de trabajo, la orientación a las necesidades del puesto y la generación de una red profesional tienen una relación positiva con la transferencia. Sin embargo la duración de la formación, el número de participantes y el número de profesores no presentan relación con la transferencia. El tiempo que el participante tarda en aplicar lo aprendido se relaciona inversamente con la transferencia.

La dimensión más relevante de cara a predecir la transferencia es la orientación a las necesidades del puesto.

\section{PALABRAS CLAVE}

Evaluación de la transferencia, evaluación de la formación, eficacia de la formación.

\section{ABSTRACT}

This paper shows the results of the evaluation of training transfer in the Spanish Public Administration with the aim of identifying the factors that influence it.

We analyzed 1475 answers and 69 courses organized by the Spanish Public Administration during the first semester of 2016. Direct transfer was evaluated through the Deferred Transfer Scale (CdE; Pineda, Quesada y Ciraso, 2011) while indirect transfer was evaluated through the Training Transfer Factors Scale (FET; Pineda, Quesada y Ciraso, 2013). 
The results show that trainee factors, organization factors, the orientation towards job requirements and the creation of a professional community all have a positive correlation with training transfer. However the duration of the training, the number of trainees and the number of instructors do not have a correlation with transfer.

The time that laps until the trainees finally apply what they learnt in the training has an inverse correlation to transfer.

The most important factor when it comes to predicting training transfer is the orientation towards job requirements.

\section{KEYWORDS}

Training transfer; training evaluation; training effectiveness.

\section{SUMARIO}

I. INTRODUCCIÓN. II. METODOLOGÍA. 1. POBLACIÓN Y MUESTRA. 2. INSTRUMENTOS. 3. PROCEDIMIENTO. III. RESULTADOS. VII. CONCLUSIONES, DISCUSIÓN Y SUGERENCIAS DE MEJORA. V. REFERENCIAS BIBLIOGRÁFICAS.

\section{INTRODUCCIÓN}

En el sector público, al igual que en el privado, la formación es un factor fundamental para incrementar la productividad de los empleados y de la organización en su conjunto (Lama, 2014).

La formación no comienza ni finaliza con la impartición de acciones formativas, es un proceso continuo en el que cobra una especial importancia la evaluación. Existen distintos modelos de evaluación de la formación, valgan como ejemplo de los mismos las revisiones realizadas por Kumpikaite (2007), Pineda (2000) y Sanduvete (2008). Seguramente el más conocido sea el de Kirkpatrick (1959), que para evaluar los resultados conseguidos con las acciones formativas plantea distintos niveles (reacción, aprendizaje, transferencia e impacto). No obstante, todos los modelos recogen el nivel de transferencia, entendida como el grado en el que los participantes aplican los conocimientos y competencias adquiridos durante la formación en su contexto de trabajo (Pereda, Berrocal, Alonso, 2014). Para que esto ocurra, «la conducta aprendida debe ser generalizada y mantenida en el puesto durante un periodo de tiempo determinado» (Baldwin y Ford, 1988: 63).

Existen distintos modelos de evaluación de la transferencia (e.g., Holton, 2005; Burke y Hutchins, 2008), en España destaca el elaborado por Pineda et al., (2013). Las autoras distinguen entre evaluación directa de la transferencia (que se centra en medir el grado de transferencia de los aprendizajes mediante el uso de técnicas e instrumentos de medida específicos que reflejen los cambios en el lugar de trabajo producidos por la formación) y la evaluación indirecta (referidos a los factores que influyen en la transferencia).

El modelo de Pineda et al. (2013) consta de ocho factores organizados en tres dimensiones: dimensión del participante, dimensión de la formación y dimensión de la organización. Además incluye el aprendizaje y la intención de transferencia como factores relevantes, en la figura 1 se incluye la representación gráfica del modelo.

Cuando se analiza la dimensión compuesta por los factores relacionados con el participante, se encuentran evidencias de relaciones positivas entre la transferencia de la formación y la satisfacción con la formación (Alliger, Tannenbaum, Bennett, Traver y Shotland, 1997; Holton, 1996, 2005; Pineda et al., 2013; Thayer y Teachout, 1995), la motivación del participante para transferir (Axtell, Maitlis y Yearta, 1997; Bates, Kauffeld y Holton, 2007; Chiaburu y Lindsay, 2008; Colquitt, LePine y Noe, 2000; Devos, Dumay, Bonami, Bates y Holton, 2007; Grossman y Salas, 2011; Hughes, 2016; Kontoghiorghes, 2004; Machin y Fogarty, 2004; Van den Bossche, Segers y Jansen, 2010), el locus de control interno o creencia del participante de que su éxito en la transferencia depende totalmente de sí mismo (Cheng y Ho, 2001; Ciraso, Espona, Quesada, Valdivia y Pineda, 2016; Colquitt et al., 2000) y el aprendizaje adquirido (Colquitt et al., 2000; Nijman, Nijhof, Wognum y Veldkamp, 2006; Pérez, 2008; Rouiller y Goldstein, 1993; Thayer y Teachout, 1995; Velada y Caetano, 2007). 


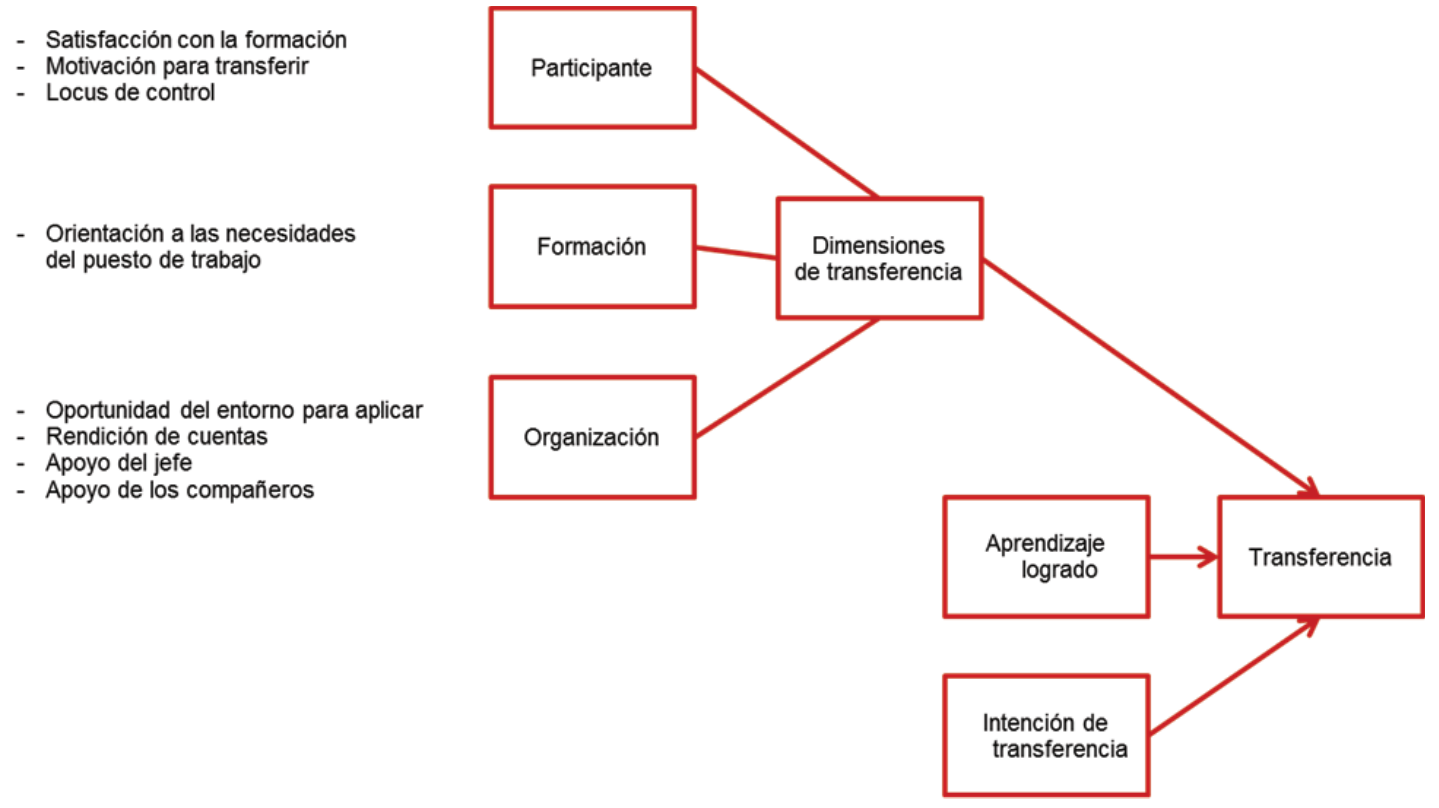

Si se analizan los factores que componen la dimensión del entorno de trabajo y la organización, también se encuentran relaciones positivas entre las posibilidades del entorno para aplicar, que según Pineda et al., (2013) se refiere a los elementos percibidos como externos al participante, como son los recursos que se le proporcionan para aplicar los aprendizajes, la carga de trabajo, los imprevistos y contratiempos y la intervención de terceras personas, que pueden condicionar la capacidad de transferir al lugar de trabajo los aprendizajes adquiridos (Clarke, 2002; Gilpin-Jackson y Bushe, 2007; Haines, 1994; Hughes, 2016; Lim y Johnson, 2002 y Pineda et al., 2013); el apoyo de jefes y compañeros para transferir (Austin, Weisner, Schrandt, Glezos-Bell y Murtaza, 2006; Awoniyi, Griego y Morgan, 2002; Blume, Ford, Baldwin y Huang, 2010; Brinkerhoff, 2006; Burke y Baldwin, 1999; Burke y Hutchin, 2008; Clarke, 2002; Cromwell y Kolb, 2004; Gilpin-Jackson y Busche, 2006; Grossman y Salas, 2011; Hawley y Barnard, 2005; Hughes, 2016; Kontoghiorghes, 2001; Saks y Belcourt, 2006; Salas y Stagl, 2015); y la rendición de cuentas de la aplicación, referida al grado en que la organización, y especialmente el superior de los empleados, pide evidencias de los resultados de la transferencia (Burke y Baldwin, 1999; Clarke, 2002; Chiaburu y Marinova, 2005; Ford, Quiñones, Sego, y Sorra, 2006; Foxon, 2008; Kontoghiorghes, 2001; Longenecker, 2004; Russ-Eft, 2002; Saks y Belcourt, 2006).

Sin embargo, no siempre ha habido coincidencia en los resultados obtenidos por los distintos autores, así en el factor apoyo de los jefes y compañeros para transferir, algunos encuentran una relación indirecta con la transferencia (e.g., Chiaburu, Van Dam y Hutchins, 2010; Nijman et al., 2006; Schindler, 2013); y otros hablan de relación débil o incluso niegan que exista tal relación (Axtell et al., 1997; Devos et al., 2007; Nijman et al., 2006; Van der Klink, Gielen y Nauta, 2001; Velada, Caetano, Michel, Lyons y Kavanagh, 2007).

Analizando la dimensión de la formación, distintos autores encuentran relaciones entre la transferencia y el factor orientación a las necesidades del puesto de trabajo (e.g., Baldwin y Ford, 1988; Bates et al., 2007; Paas y Van Merriënboer, 1994; Pérez, 2008; Rodriguez, 2005; Velada et al., 2007). Además de los factores mencionados se han encontrado relaciones entre variables que no aparecen recogidas en el modelo de Pineda et al. (2013), como son el factor generación de una comunidad o red de profesionales, referido al grado en que los participantes generan una red profesional para comentar, colaborar o compartir ideas relacionadas con la formación, la organización o su práctica profesional (e.g., Boud y Middleton, 2003; Ingvarson, Meiers y Beavis, 2005). La transferencia también aparece relacionada con la variable tiempo; por ejemplo, algunos estudios han destacado el impacto del momento en el que tiene lugar la formación (Broad, 2005; Salas, Tannenbaum, Kraiger y Smith-Jentsch, 2012) y han puesto de manifiesto que el uso de habilidades aprendidas en la formación declina con el paso del tiempo (e. g., Blume et al., 2010; Tziner y Falbe, 1993), pero no existe un índice formalizado que ilustre cómo el constructo cambia en función del paso del tiempo. 
Algunos estudios sostienen que el declive comenzaría dos meses después de la formación (Saks, 2002; Saks y Belcourt, 2006), otros que se produciría al año (Arthur, Bennett, Stanush y McNelly, 1998) y otros, incluso, que se produciría a los siete años (Siassakos, Fox, Crofts, Hunt, Winter y Draycott, 2011). Saks y Belcourt (2006) encontraron que las puntuaciones en transferencia descendían un $50 \%$ al medirlas un año después de la formación.

Teniendo en cuenta otros factores relacionados con la dimensión de la formación, los autores no encuentran relaciones con la transferencia; por ejemplo, en la duración en horas de la formación (Ingvarson et al., 2005).

A la vista de la revisión bibliográfica realizada y en línea con las conclusiones de Valencia-Rodríguez (2013), parece evidente que no existe un acuerdo sobre una taxonomía o modelo único de factores de transferencia. Tampoco parece factible que vaya a dibujarse un modelo único aplicable a todos los contextos, ya que cada uno es diferente y el ámbito organizativo (ya sea público o privado), evoluciona rápidamente. Estas diferencias contextuales dificultan la comparación de resultados obtenidos por los distintos autores en el ámbito de la transferencia de la formación.

En la administración pública española se han llevado a cabo distintos estudios con el fin de evaluar la transferencia de la formación, destacan el estudio ETAPE (Pineda, Quesada, Espona, Ciraso y García, 2012) y el Modelo de Evaluación del eLearning en la Administración Pública (Ciraso et al., 2016). Los resultados del estudio ETAPE ponen de manifiesto que las características de la formación que influyen en la transferencia son aquellas que están relacionadas con las necesidades del puesto, que generaran una reacción positiva en los participantes, aquellas en las que los participantes creen que depende de ellos aplicar lo aprendido, en las que cuentan con un entorno laboral que facilita la transferencia y en las que existe un proceso que permite a los participantes rendir cuentas a sus superiores sobre lo aprendido. Además, los resultados indicaron que la formación presencial era más eficaz que la formación online.

En este artículo se recoge parte de los resultados encontrados en el proyecto «Evaluación de la eficacia de la formación en la Administración Pública: la transferencia al puesto de trabajo» financiado por el INAP (Alonso, Berrocal, González-Ortiz y Centolosi, 2017), cuyo objetivo general era analizar la transferencia de la formación del INAP con el fin de conocer los factores que la influencian en mayor medida.

Tomando en cuenta los resultados encontrados en la revisión teórica se plantearon los siguientes objetivos específicos:

1. Analizar si los factores de los participantes se relacionan con la transferencia.

- Hipótesis 1: Los factores de los participantes a) satisfacción con la formación, b) locus de control interno, c) motivación para transferir y d) nivel de aprendizaje adquirido se relacionan de forma positiva con la transferencia.

2. Analizar si los factores del lugar de trabajo y/o la organización se relacionan con la transferencia.

- Hipótesis 2: Los factores del lugar de trabajo y/o la organización a) rendición de cuentas, b) posibilidades del entorno para la aplicación, c) apoyo de los compañeros y del jefe para transferir se relacionan de forma positiva con la transferencia.

3. Analizar si los factores de la formación se relacionan con la transferencia.

- Hipótesis 3: La orientación a las necesidades del puesto de trabajo se relaciona de forma positiva con la transferencia.

- Hipótesis 4: La duración de la formación en horas no se relaciona con la transferencia.

- Hipótesis 5: El número de participantes en la formación y el número de profesores no se relacionan con la transferencia.

- Hipótesis 6: La generación de una comunidad o red profesional se relaciona de forma positiva con el nivel de aprendizaje adquirido.

- Hipótesis 7: La generación de una comunidad o red profesional se relaciona de forma positiva con la transferencia.

4. Analizar la relación de la transferencia con el tiempo en aplicar el aprendizaje al puesto.

- Hipótesis 8: El tiempo que tarde en aplicarse lo aprendido en el curso de formación tiene una relación inversa con la transferencia: a menor tiempo de aplicación mayor transferencia. 
5. Detectar las variables que mejor predicen la transferencia de la formación al puesto de trabajo al finalizar la formación.

- Hipótesis 9. La variable recogida al finalizar la formación que más peso tiene a la hora de explicar la transferencia es la orientación a las necesidades del puesto.

\section{METOdOLOGÍA}

\section{Población y muestra}

La muestra estaba formada por 62 acciones formativas y 69 cursos (cinco acciones formativas tuvieron dos ediciones y una acción tuvo tres). La modalidad de impartición de los cursos fue muy similar, con un $49.3 \%$ (34) de cursos presenciales y un $50.7 \%$ (35) de cursos online.

La media de participantes por curso fue de 39.7 alumnos y la desviación estándar de 18.4. La media de participantes en la modalidad online (57.89) fue muy superior a la media de participantes en modalidad presencial (20.94), siendo la media de horas muy similar para las dos modalidades (28.29 horas de duración media para la modalidad online y 23.53 para la presencial).

Con relación al número de profesores que participaron por curso, varía en función de distintos criterios, como puede ser la duración del curso, los temas a abordar, etc. Participando uno, dos o tres profesores en el $79.7 \%$ de las acciones formativas.

La muestra estuvo compuesta por 1.475 respuestas válidas, provenientes de los participantes en 69 cursos, de los cuales 803 eran mujeres (54.4\%) y 668 eran varones (45.3\%). La edad media fue de 46.40 años y la antigüedad media en su puesto de trabajo era de 9.4 años.

El 91.2\% de los trabajadores realizó un curso de formación a lo largo del periodo de recogida de datos, el $8.6 \%$ realizó dos cursos y solamente hubo tres personas que realizaron $3(0.2 \%)$.

Los participantes eran trabajadores de la administración autonómica $(4,1 \%)$, local $(3,5 \%)$ y el resto de la Administración General del Estado (AGE), con una representación de los distintos organismos.

Con relación al nivel educativo de la muestra, $59.5 \%$ indicó tener estudios universitarios, el $28 \%$ había realizado hasta el bachillerato o formación profesional y el 10\% había completado estudios de postgrado (máster y/o doctorado).

En la muestra aparecieron representados todos los niveles de la AGE (del 14 al 30). El grupo con mayor representación fue el formado por los niveles del 14 al 24 , con un $74.4 \%$ de la muestra; mientras que el grupo que incluía los niveles del 25 al 30 constituyó un 19.1\%. El 30.9\% de los participantes indicó tener personas a su cargo.

En cuanto a los motivos expresados por los participantes para realizar la acción formativa, cabe destacar que el $94.8 \%$ indicó que había sido por iniciativa propia y únicamente el $2.5 \%$ consideró que fue animado por su jefe.

La mayor parte de las acciones formativas se realizaron dentro de la jornada laboral (44.0\%) o de forma mixta, incluyendo horas tanto dentro como fuera de la jornada laboral (32.1\%).

Las 1.475 respuestas válidas recogidas corresponden al $53.9 \%$ de los participantes en los cursos incluidos en el estudio y que cumplimentaron el primer cuestionario a la finalizaron la acción formativa. De ellas, cumplimentaron el segundo cuestionario a los cuatro meses de la finalización del curso el $49.6 \%$; es decir, 731 participantes. Con respecto a los profesores / tutores cumplimentaron el cuestionario 121 de los 177 posibles $(68.4 \%)$.

\section{Instrumentos}

Al finalizar la acción formativa se aplicaron los siguientes instrumentos:

El cuestionario de Factores de Eficacia de la Transferencia (FET), de Pineda et al. (2013). Este cuestionario incluye 42 ítems que evalúan tres dimensiones y siete factores:

- Dimensión del participante, que incluye los factores de satisfacción con la formación, locus de control interno y motivación para transferir.

- Dimensión del entorno de trabajo, que incluye los factores de rendición de cuentas, posibilidades del entorno para la aplicación y apoyo para transferir.

- Dimensión de la formación, que incluye el factor orientación a las necesidades del puesto de trabajo. 
Junto con el cuestionario FET se incluyeron tres ítems que medían el factor aprendizaje.

El tipo de escala utilizada en las preguntas cerradas era tipo Likert de cinco anclajes en la que el uno significaba totalmente en desacuerdo y el cinco totalmente de acuerdo.

Cuando habían pasado cuatro meses desde que los participantes habían finalizado la formación se les aplicó el Cuestionario de Eficacia de la Formación (CdE) de Pineda et al., 2011), que mide el grado de transferencia desde la perspectiva del participante. El objetivo del cuestionario es identificar el grado de aplicación en el puesto de trabajo de lo aprendido por los participantes en la formación.

Para conocer en qué medida los participantes generaron una red profesional con la que compartir los conocimientos adquiridos en el curso, se crearon dos ítems que había que responder utilizando una escala tipo Likert: «después de finalizar el curso he comentado, colaborado o compartido ideas relacionadas con la formación y la práctica profesional con otros participantes del curso» y "algunos de los aprendizajes adquiridos en el curso los he compartido con otras personas del entorno profesional».

Con respecto al tiempo que se tarda desde que finaliza el curso hasta que se comienzan a aplicar los aprendizajes se evaluó a través de la pregunta: ¿cuánto tiempo has tardado en aplicar lo aprendido en el curso que realizaste a tu puesto de trabajo? Las opciones de respuesta de esta variable eran las siguientes: 5: inmediatamente; 4: antes de un mes; 3: de uno a tres meses; 2: de tres a seis meses; 1: más de seis meses.

\section{Procedimiento}

En primer lugar se procedió, de forma conjunta con la Subdirección de Formación del INAP, a analizar las fases del estudio de evaluación de la transferencia que se iba a seguir, estableciendo como criterios para incluir las distintas acciones formativas en la investigación que fueran acciones de entrenamiento o de capacitación; esto es, que estuvieran dirigidas al desarrollo o adquisición de conocimientos y competencias directamente relacionadas con el trabajo actual o futuro de los participantes; que fueran a impartirse durante el primer semestre de 2016, con el fin de facilitar la recogida de información de la transferencia antes de la finalización del año. No se incluyeron en el proyecto aquellas acciones consideradas informativas o de sensibilización, o en las que no estaban delimitados el tipo de participantes o los objetivos.

Para llevar a cabo la selección de las acciones formativas incluidas en el proyecto, se realizó el análisis del plan de formación del primer semestre, con el fin de asegurar que las acciones elegidas cumplían los criterios establecidos.

Se realizó un calendario de recogida de datos en el que se incluía el detalle de las acciones formativas sobre las que se iba a realizar la recogida de información y se definió el proceso de trabajo a seguir en función de la modalidad de la acción formativa: presencial, semi-presencial o e-learning.

La recogida de datos en las acciones presenciales y semi-presenciales se haría a la finalización de las mismas y en formato de papel y lápiz. En las acciones de e-learning se haría online, a través de un cuestionario vía web.

Transcurridos cuatro meses desde la finalización de la acción formativa se enviaba un correo electrónico a los participantes que habían cumplimentado el primer cuestionario, en el que se incluía el link a la web en la que estaba disponible el instrumento de evaluación de la transferencia.

\section{RESULTADOS}

La variable dependiente de este estudio es la transferencia de la formación. La transferencia media obtenida es de 2.49 puntos en una escala de cinco puntos y la desviación estándar es de.81.

La variable transferencia es una puntuación global calculada tras la aplicación del cuestionario CdE de Pineda et al. (2011). De forma previa a la comprobación de las hipótesis planteadas, se realizaron los análisis previos para contrastar los supuestos de normalidad y homogeneidad de varianzas.

Para ver el grado de relación entre la variable criterio y las variables predictoras relacionadas con los factores del cuestionario FET se calcularon las correlaciones de Pearson. Las correlaciones entre la puntuación global de transferencia (ver tabla 1) y los factores de los participantes son positivas y significativas (entre.322 y.374), lo que permite aceptar la hipótesis 1.

De la misma forma, los factores del entorno de trabajo se relacionan de forma positiva con la transferencia, tal y como se observa en la matriz de correlaciones, por lo que se acepta la hipótesis 2. 
También existen relaciones significativas, elevadas y positivas entre la puntuación total de transferencia y el factor orientación a las necesidades del puesto, lo que permite afirmar que se cumple la Hipótesis 3 . Destacan las altas correlaciones de la variable orientación a las necesidades del puesto con el resto de factores.

TABLA 1. CORRELACIONES DE PEARSON ENTRE LOS FACTORES DEL LUGAR DE TRABAJO Y/O LA ORGANIZACIÓN Y LA TRANSFERENCIA

\begin{tabular}{|c|c|c|c|c|c|c|c|c|c|c|c|}
\hline & & M & SD & Transfer & 1 & 2 & 3 & 4 & 5 & 6 & 7 \\
\hline 1. & Satisfacción con la formación & 3.84 & .82 & $.322^{* *}$ & 1 & & & & & & \\
\hline 2. & Motivación para transferir & 4.07 & .63 & $.374^{* *}$ &, $52^{* *}$ & 1 & & & & & \\
\hline 3. & Locus de control interno & 2.99 & 1.02 & $.333^{* *}$ & $19^{* *}$ &, $36^{* *}$ & 1 & & & & \\
\hline 4. & Nivel de Aprendizaje Adquirido & 3.55 & .79 & $.358^{* *}$ &, $72^{* *}$ &, $41^{* *}$ &, $18^{* *}$ & 1 & & & \\
\hline 5. & $\begin{array}{l}\text { Posibilidades del entorno para } \\
\text { aplicar }\end{array}$ & 3.72 & .68 & $.258^{* *}$ & 0,02 &, $08^{* *}$ &, $32^{* *}$ & 0,04 & 1 & & \\
\hline 6. & Apoyo para transferir & 2.92 & .89 & $.341^{* *}$ &, $20^{* *}$ &, $29^{* *}$ &, $29^{* *}$ &, $19^{\star \star}$ & $31^{* *}$ & 1 & \\
\hline 7. & $\begin{array}{l}\text { Rendición de cuentas de la } \\
\text { aplicación }\end{array}$ & 2.11 & .76 & $.364^{* *}$ &, $15^{* *}$ &, $17^{* *}$ &, $21^{* *}$ &, $18^{* *}$ &, $11^{* *}$ &, $66^{* *}$ & 1 \\
\hline 8. & $\begin{array}{l}\text { Orientación a las necesidades } \\
\text { del puesto de trabajo }\end{array}$ & 3.14 & .85 & $.577^{* *}$ &, $56^{* *}$ &, $56^{* *}$ &, $43^{* \star}$ &, $49^{* *}$ &, $22^{* *}$ &, $42^{* *}$ &, $39^{* *}$ \\
\hline
\end{tabular}
${ }^{* \star} p<.001$

Con respecto a la duración de la formación y el número de participantes no se han encontrado relaciones con la transferencia, probablemente porque ambas variables son muy similares en las distintas acciones formativas incluidas en el estudio (ver tabla 2). Por otro lado, la relación entre el número de profesores y la transferencia encontrada es muy pequeña como para tenerla en cuenta. Por tanto, se aceptan las hipótesis 4 y 5 .

TABLA 2. CORRELACIONES DE PEARSON ENTRE LOS FACTORES DEL LUGAR DE TRABAJO Y/O LA ORGANIZACIÓN Y LA TRANSFERENCIA

\begin{tabular}{llcccc}
\hline & & Transfer & $\mathbf{1}$ & $\mathbf{2}$ & $\mathbf{3}$ \\
\hline 1. & Horas del curso & -.063 & $-.097^{* *}$ & 1 & \\
\hline 2. Número de participantes & .016 & $-.114^{* *}$ & $-.081^{* *}$ & 1 \\
\hline 3. & Número de profesores & $.080^{*}$ & .010 & $.077^{* *}$ & $.106^{* *}$ \\
\hline
\end{tabular}

${ }^{*} p<.05 ;{ }^{* *} p<.001$

Tal y como se puede comprobar en la tabla 3, compartir ideas con otros participantes del curso y con otras personas del entorno profesional se relaciona de forma positiva con la trasferencia de la formación, especialmente la segunda con una correlación de.539 entre ambas variables. En la tabla también se muestran las relaciones de ambas variables con el aprendizaje, que aunque moderadas, también son significativas, por lo que se confirman las Hipótesis 6 y 7.

TABLA 3. CORRELACIONES DE PEARSON ENTRE COMPARTIR CON OTROS PARTICIPANTES DEL CURSO, CON OTRAS PERSONAS DEL ENTORNO PROFESIONAL, LA TRANSFERENCIA Y EL APRENDIZAJE

\begin{tabular}{|c|c|c|c|c|c|}
\hline & $\mathbf{M}$ & DT & Transfer & Aprendizaje & 1 \\
\hline $\begin{array}{l}\text { 1. Comentar, colaborar o compartido ideas } \\
\text { relacionadas con la formación y la práctica } \\
\text { profesional con otros participantes del curso }\end{array}$ & 1.97 & 1.16 & $.323^{* *}$ & $.13^{* *}$ & 1 \\
\hline $\begin{array}{l}\text { 2. Compartir aprendizajes con otras personas } \\
\text { del entorno profesional }\end{array}$ & 2.79 & 1.17 & $.539^{* *}$ & $.29^{* *}$ & $.421^{* *}$ \\
\hline
\end{tabular}


GAPP. Nueva Época - N.17, mayo 2017 - ISSN: 1989-8991 - DOI: 10.24965/gapp.v0i17.10405 - [Págs. 113-127]

Evaluación de la eficacia de la formación en la Administración Pública: la transferencia al puesto Aitana González-Ortiz de Zárate / Miguel Aurelio Alonso García / Francisca Berrocal Berrocal

Los resultados indican que cuanto más próximo esté en el tiempo la aplicación de los aprendizajes al puesto de trabajo, mayor transferencia se produce, por tanto, se cumple la Hipótesis 8 (ver tabla 4).

\section{TABLA 4. CORRELACIONES DE PEARSON ENTRE EL TIEMPO TRANSCURRIDO ENTRE LA FINALIZACIÓN DEL CURSO Y LA TRANSFERENCIA}

\begin{tabular}{|c|c|c|c|}
\hline & M & DT & Transfer \\
\hline $\begin{array}{l}\text { Tiempo transcurrido entre la finalización del curso y la aplicación de los aprendizajes } \\
\text { al puesto de trabajo }\end{array}$ & 3.51 & 1.57 & $.565^{\star *}$ \\
\hline
\end{tabular}

${ }^{* *} \mathrm{p}<.001$

Para conocer qué variables de las recogidas cuando finaliza la formación (a los participantes y al formador) explican mejor la transferencia al puesto de trabajo se realizó un análisis de regresión.

Se tomó como variable criterio la transferencia de la formación y cómo variables predictoras las dimensiones del FET y las percepciones de los participantes en la acción formativa, nada más finalizar, sobre el aprovechamiento del curso y las posibilidades de transferencia.

En concreto, se intenta conocer en qué medida se puede predecir la transferencia de una acción formativa preguntando a los participantes cuando esta finaliza.

Se realizó un análisis de regresión múltiple por pasos incluyendo las variables relacionadas con el participante, el entorno y la formación. Los resultados ponen de manifiesto que la variable orientación a las necesidades del puesto de trabajo explica el $35 \%$ de la información. A medida que se van añadiendo variables el $\mathrm{R}^{2}$ incrementa hasta el $42 \%$ con las variables que se presentan en la tabla 5 . Por otro lado, el valor del estadístico de Durbin-Watson (1.91) es mayor a 1.4, lo que señala que tanto los residuos como las observaciones de las variables utilizadas en el análisis de regresión son independientes.

TABLA 5. RESUMEN DEL MOdELO DE REGRESIÓN LINEAL POR PASOS CON LAS VARIABLES RECOGIDAS AL FINALIZAR LA FORMACIÓN

\begin{tabular}{|c|c|c|c|c|c|c|c|}
\hline Modelo & Variable & $\mathbf{R}$ & $\begin{array}{c}\mathbf{R}^{2} \\
\text { ajustado }\end{array}$ & $\begin{array}{l}\text { Error estándar de } \\
\text { la estimación }\end{array}$ & Cambio de $\mathbf{R}^{2}$ & $\mathbf{F}$ & $\begin{array}{c}\text { Coeficientes } \\
\text { estandarizados }(\beta)\end{array}$ \\
\hline 1 & ONP & .589 & .35 & .76 & $.347^{* *}$ & 325.80 & .36 \\
\hline 2 & RCA & .612 & .37 & .75 & $.028^{* *}$ & 26.95 & .17 \\
\hline 3 & TIEM & .629 & .39 & .74 & $.02^{* *}$ & 20.66 & .12 \\
\hline 4 & $\mathrm{LoCl}$ & .639 & .41 & .73 & $.014^{* *}$ & 14.18 & .15 \\
\hline 5 & CONO & .649 & .42 & .72 & $.013^{* *}$ & 13.64 & .13 \\
\hline
\end{tabular}

${ }^{*} p<.05 ;{ }^{* *} p<.001$

ONP: Orientación a las necesidades del puesto de trabajo; RCA: Rendición de cuentas de la aplicación; TIEM: Tiempo estimado en aplicar lo aprendido al puesto de trabajo; LoCl: Locus de control interno; CONO: Nivel de conocimientos adquiridos o desarrollados en el curso.

Por tanto, se cumple la hipótesis 9, debido a que entre las variables recogidas al finalizar la formación, la que más peso tiene a la hora de explicar la transferencia es la orientación a las necesidades del puesto.

\section{CONCLUSIONES, DISCUSIÓN Y SUGERENCIAS DE MEJORA}

La satisfacción y el aprendizaje son dos niveles de evaluación que están presentes en los distintos modelos de evaluación de la formación, y en nuestro estudio aparecen como factores clave para facilitar la transferencia. Con respecto a la satisfacción con la formación, se ha encontrado una correlación de.322 con la transferencia, resultado que es coherente con algunos de los estudios previos (e.g. Alliger et. al., 1997; Pineda et al., 2013). Además, cuando un participante está satisfecho con la formación y con el formador/ tutor es más probable que esté más abierto a la adquisición o desarrollo de nuevos aprendizajes, por lo que 
es lógico que se haya encontrado una correlación muy alta entre estos dos factores ( $r=.723)$, como encuentran otros autores (e.g., Cabrera, 1993; Pineda, 2002); así como que muestren una mayor motivación para aplicarlos posteriormente, habiéndose encontrado también una alta correlación entre la satisfacción y la motivación para transferir $(r=.520)$.

Estos resultados remarcan la importancia de conseguir una elevada satisfacción de los participantes, por lo que es recomendable continuar evaluando este factor en la totalidad de los cursos del INAP, tal y como ya se está haciendo, y tener en cuenta los resultados y sugerencias indicadas por los participantes para el diseño de los planes de formación, aspecto que facilitaría la mejora continua del proceso de formación, así como atender a las necesidades e intereses los participantes, ya que estos son uno de los clientes clave de la función de la formación.

El factor aprendizaje presenta una relación positiva y significativa con la transferencia $(r=.358)$, muy similar a la encontrada por Colquitt et al. (2000). Sin aprendizaje no puede haber transferencia, ya que no se pueden producir cambios en el comportamiento de los participantes en sus puestos de trabajo si previamente no se han adquirido las competencias que lo posibiliten (Colquitt et al., 2000; Mathieu et al. 1992; Velada y Caetano, 2007; Warr y Bunce, 1995), de ahí la importancia de este factor. Sin embargo, que exista aprendizaje no implica que éste se vaya a aplicar, debido a que pueden existir distintos motivos que lo dificulten, tanto relacionados con el participante como con el entorno.

Debido a la importancia que tiene la evaluación del aprendizaje sería necesario evaluar aquellas acciones formativas del plan de formación que persigan en sus objetivos la adquisición o desarrollo de conocimientos y competencias aplicables al puesto de trabajo. Esta evaluación, previa a la evaluación de la transferencia, aseguraría que el aprendizaje se ha producido y permitiría descartar que niveles bajos en transferencia se deban a este factor. En la actualidad, en el INAP, la evaluación del aprendizaje se realiza de forma sistemática en la formación en modalidad e-learning, y en algunas que se desarrollan en modalidad presencial. Para que la evaluación del aprendizaje se amplíe a las acciones presenciales es necesario implicar a los formadores, tanto internos como externos, debido a que generalmente son ellos los encargados de la aplicación de los instrumentos de evaluación durante la impartición del curso. También es necesario que las evaluaciones no se realicen únicamente sobre el componente de los conocimientos de las competencias, y se incluyan pruebas profesionales y situacionales, con el fin de evaluar todos los componentes y poder hacer estimaciones de las facilidades o dificultades que podrán tener los individuos para transferir, y de esta forma poder desarrollar planes de mejora individualizados de vuelta al puesto de trabajo.

En este punto es preciso insistir sobre la importancia de desarrollar planes de mejora individualizados por parte de los participantes a la finalización de la acción formativa, como medida de intervención para fomentar la transferencia (Foxon y Foxon, 1987; Hollenbeck y Ingols, 1990). La elaboración de estos planes de mejora implican, por ejemplo, que el participante haga una reflexión sobre los objetivos alcanzados, cómo va a aplicar los aprendizajes al trabajo, los medios o ayudas que va a necesitar y defina las tareas a realizar. Con su elaboración se persigue aumentar el compromiso de los participantes y su motivación. Por tanto, sería necesario que el diseño de las acciones formativas incluyera el espacio necesario para el desarrollo de planes de mejora, ya que para que realmente sea eficaz debería realizarse a la finalización de la acción formativa, como una actividad más de la misma.

Es importante remarcar la importancia del factor motivación para transferir, debido a que se ha encontrado una relación positiva y significativa con la transferencia, en línea con Bates et al. (2007), Hughes (2016), Machin y Fogarty (2004) y Van den Bossche et al. (2010). Tras el análisis de estos resultados surge la siguiente pregunta, ¿qué se puede hacer desde el área de formación para que las personas se motiven en la aplicación de lo aprendido en su puesto de trabajo? Sería necesario; por un lado, hacer partícipes a los participantes en la detección de sus propias necesidades formativas, al estar los niveles de motivación relacionados con la satisfacción de las necesidades de cada individuo y sus expectativas, aspecto que es contemplado ya por el INAP, debido a que los propios trabajadores pueden solicitar participar en aquellas acciones que están acordes a su nivel y tipo de trabajo, así como en aquellas que son de carácter transversal y; por otro lado, implicarles en la evaluación de la transferencia para que sean conscientes de los beneficios que ha tenido la formación para la mejora de su desempeño. Es probable que esta implicación de los participantes sea la razón por la que expresan una motivación para transferir muy alta (4.07 sobre 5).

Los participantes que tienen un locus de control interno alto sobre las posibilidades de transferir lo aprendido al puesto de trabajo, tienen la creencia de que el éxito en la transferencia depende de sí mismos, y eso hace que tengan una alta motivación para transferir. Quizá podrían potenciarse las expectativas de éxito 
acerca de aplicar lo aprendido al puesto a través de políticas de recursos humanos adecuadas por parte de la Administración: la implicación del jefe directo en motivar a sus colaboradores para asistir a determinadas acciones formativas, la generación de expectativas antes de asistir a la formación, el apoyo posterior cuando ha realizado el curso para que lo aplique, etc.

En definitiva, los factores de los participantes están relacionados con la transferencia, y además existe relación entre satisfacción y aprendizaje, la motivación para transferir se relaciona con ambas y a su vez con el locus de control.

En cuanto a los factores del lugar de trabajo y/o la organización se ha encontrado una relación positiva con la transferencia, en concreto, los relacionados con la rendición de cuentas, las posibilidades del entorno para la aplicación y el apoyo para transferir; lo que implica que compañeros y jefes apoyan en la transferencia y además solicitan información para conocer los resultados.

Estos resultados ponen de manifiesto la importancia de que la organización y, en especial el superior inmediato del participante, solicite evidencias de la efectividad de la transferencia, lo que reflejaría su preocupación por los resultados de la formación y por las mejoras en el desempeño del colaborador. Esta rendición de cuentas se puede realizar de diversas formas como, por ejemplo, la realización de un informe sobre los aspectos más relevantes de la formación, la realización de una presentación con los contenidos básicos a compañeros y mandos, o la inclusión de la formación dentro de los objetivos de desempeño y desarrollo de los individuos (Longenecker, 2004), lo que estaría reflejando que la organización realmente considera la formación como una inversión, al estar alineada con los objetivos a alcanzar, y estaría recompensando los esfuerzos extra que hacen las personas para mejorar de forma continua en su trabajo. Este tipo de actuaciones se han relacionado con un aumento de la motivación y de la percepción de utilidad de los programas (Colquitt et al., 2000).

Asimismo, es preciso que el trabajador reciba el apoyo necesario para aplicar los nuevos aprendizajes, tanto por parte de sus compañeros como por parte de su superior, debiendo proporcionar éste el apoyo emocional y los recursos necesarios. Para que esto suceda, se debe implicar a los mandos en la detección de las necesidades formativas de sus colaboradores; por ejemplo, llama la atención que únicamente el 2,5\% de los participantes indiquen que su jefe les animó a realizar la formación, si bien el INAP requiere que los mandos validen la formación solicitada por sus colaboradores. Es posible que no sea suficiente y que fuera útil que desde la unidad de formación se solicitara información directa a los mandos y directivos sobre las necesidades detectadas en sus direcciones y equipos de trabajo. El estudio de detección de necesidades formativas debería hacerse periódicamente, normalmente de forma anual, con la posibilidad de recibir peticiones directas a lo largo de todo el periodo, para que puedan contemplarse aquellas necesidades que surjan de forma imprevista. En este punto, nos gustaría destacar de forma muy positiva, el bajo porcentaje de participantes que indican que uno de los motivos de no transferir es que no tiene el suficiente apoyo de las personas con las que trabaja $(6 \%)$.

Desde nuestro punto de vista este apoyo se debería extender más allá de jefes y compañeros e incluir en la planificación de la formación (en aquellas acciones consideradas estratégicas o claves) el apoyo por parte de formadores / tutores durante un plazo de tiempo posterior a la finalización de la acción formativa. De esa forma el participante podría solicitar ayuda en el caso de encontrar dificultades a la hora de transferir los aprendizajes a su trabajo.

La orientación a las necesidades del puesto de trabajo se relaciona de forma positiva con la transferencia. Se obtuvo una correlación muy alta entre este factor y la transferencia, lo que lleva a considerar que es uno de los factores más importantes. Este resultado está en la línea con los obtenidos por distintos autores (Bates et al., 2007; Pérez, 2008; Rodríguez, 2005; Velada et al., 2007), considerando que la obtención de puntuaciones bajas implicaría dificultades en la transferencia (Lama, 2014). Además, existe una alta relación entre la orientación a las necesidades del puesto y el resto de factores, lo que lo convierte en un factor central en todo el proceso de formación, con influencia en todos los demás. Con respecto a este factor encontramos una semejanza con el factor de aprendizaje, ya que si bien el hecho de que el contenido de la formación se relacione con el puesto de trabajo del participante no garantiza la transferencia, sí es un facilitador clave de la misma.

El factor orientación a las necesidades del puesto indica la importancia de hacer una buena detección de necesidades, que permita determinar qué problemas de desempeño pueden ser subsanados a través de formación, o que aspectos del mismo pueden ser potencialmente mejorados, al mismo tiempo que se garantiza la relación directa entre el contenido de la formación y el trabajo desempeñado por los participan- 
tes. Asimismo, implica diseñar las acciones formativas de tal forma que se facilite la transferencia. Cuanto más cercanos estén los contenidos, actividades, materiales didácticos, ejemplos, etc. utilizados durante la formación a la realidad laboral de los participantes, mayor facilidad tendrán éstos para transferir los nuevos comportamientos a su trabajo, mejor será su percepción de utilidad y su motivación hacia la transferencia. No tener en cuenta este factor en la elaboración del plan de formación y en el diseño de las acciones formativas, además de afectar a la satisfacción de los clientes de la función de formación (participantes, mandos y directivos, etc.), implicaría que la formación realmente sería un gasto y no una inversión para lograr la mejora continua de la organización y de las personas que la conforman, al poder estar adquiriendo los participantes conocimientos y competencias poco o nada relevantes para su trabajo.

No existen relaciones entre los factores de formación (duración del curso, número de participantes y número de profesores) y la transferencia. Estas variables han sido muy poco estudiadas; por ejemplo, en la revisión bibliográfica realizada no se han encontrado investigaciones en las que se analizaran las variables de número de participantes y número de profesores. Respeto al número de horas, Ingvarson et al. (2005) tampoco encontraron diferencias significativas. Es preciso tener en cuenta que la mayor parte de las acciones del estudio tenían una duración de entre 15 y 50 horas, y que contaban con un número de participantes y profesores similar. Cabe esperar que estudios que incluyan formaciones con una variabilidad mayor en cuanto a horas o número de participantes, encuentren diferencias.

Por otro lado, se han encontrado relaciones significativas entre la generación de una comunidad o red profesional y el nivel de aprendizaje adquirido. De la misma forma, la generación de una comunidad o red profesional se relaciona de forma positiva con la transferencia, relación que resulta significativa. Este resultado es interesante y estaría indicando la importancia de aumentar el uso de redes profesionales, algunas de ellas ya se utilizan en la Administración y los trabajadores pueden acceder y participar en las mismas, haciendo una mayor difusión de los aprendizajes adquiridos. De una forma más concreta, sería recomendable planificar momentos y espacios en los que, finalizada la formación, los participantes de la acción formativa pudieran compartir sus experiencias sobre la aplicación de los aprendizajes a sus puestos de trabajo, por ejemplo, planificando una o más sesiones en las que pudieran analizar los beneficios obtenidos, las dificultades y cómo se han solventado, etc. Para asegurar que esta práctica se realiza, sería necesario que en el diseño e impartición de las acciones formativas estuvieran contempladas estas sesiones como una parte más del curso y no como una posible actividad voluntaria, ya que lo que no se planifica y no se presupuesta, difícilmente se lleva a cabo. Esta práctica conseguiría una doble finalidad; por un lado, facilitar a la organización la realización de un seguimiento de la evolución de la transferencia, favoreciendo su afianzamiento y desarrollando planes de mejora en caso de ser necesario y; por otro lado, proporcionando oportunidades para comentar, colaborar o compartir ideas relacionadas con la formación, la organización o su práctica profesional a los participantes. En esta línea, los participantes pueden compartir los aprendizajes con sus compañeros, realizando, por ejemplo, presentaciones de las principales ideas y posibles aplicaciones del aprendizaje adquirido.

Respecto a la relación de la transferencia con el tiempo tardado en aplicar el aprendizaje, los resultados obtenidos establecen una relación inversa y significativa entre tiempo y transferencia. En otras palabras, cuanto más cercano esté en el tiempo la aplicación de los nuevos comportamientos al trabajo, mayor será la transferencia.

Este resultado tiene importantes implicaciones de cara a la planificación temporal de las acciones formativas, siendo necesario que la impartición de las mismas estén lo más cercanas posible al momento en el que el trabajador tiene que aplicar los nuevos comportamientos a su trabajo, esto es fundamental cuando los objetivos de la formación son el desarrollo de competencias técnicas frente a las competencias genéricas, debido a que las primeras están más sujetas al olvido si no se ponen en práctica de una forma relativamente rápida.

Por último, se planteaba como objetivo detectar las variables que mejor predicen la transferencia de la formación al puesto nada más finalizar la formación. Las variables que mejor permiten predecir la transferencia son: orientación a las necesidades del puesto de trabajo; rendición de cuentas de la aplicación; tiempo estimado en aplicar lo aprendido al puesto de trabajo; locus de control interno; nivel de conocimientos adquiridos o desarrollados en el curso. Estas variables permiten explicar el $42 \%$ de la variable dependiente, valor superior al conseguido por autores como Pineda et al. (2013), y sólo orientación a las necesidades del puesto explica el 35\% (Quesada, 2014, también encontró que esta variable era la que tenía mayor peso).

Los resultados obtenidos en este estudio implican la importancia de que la formación responda a necesidades concretas del puesto de trabajo de los participantes, tanto actuales como futuras. Sin embargo, el 
proceso de detección de necesidades no debería implicar solo a trabajadores y a sus superiores inmediatos, ya que esta forma de actuar solamente permitiría detectar necesidades de formación de carácter inmediato; esto es, las derivadas de problemas que se hayan detectado en el desempeño del trabajo. Por tanto, resulta necesario complementar estas necesidades con las estratégicas y que se derivan de los objetivos y plantes estratégicos de la Administración, permitiendo de esta forma actuar proactivamente a la hora de anticipar las necesidades, al mismo tiempo que alinea los objetivos de la organización con los de la formación. El órgano responsable de definir estas necesidades estratégicas sería el área de formación del INAP, tal y como ya se está haciendo.

Si bien, una adecuada detección de necesidades es clave, no es suficiente siendo necesario planificar, diseñar, impartir y evaluar la formación teniendo como foco facilitar la transferencia, así como tener en cuenta el plazo temporal en el que los participantes podrán aplicar los nuevos comportamientos a sus puestos de trabajo.

\section{REFERENCIAS BIBLIOGRÁFICAS}

ALLIGER, G. M., TANNENBAUM, S. I., BENNETT, W., TRAVER, H. y SHOTLAND, A. (1997). "A meta-analysis of the relations among training criteria", Personnel psychology, 50(2), 341-358. DOI: 10.1111/j.1744-6570.1997. tb00911.x.

ALONSO, M. A., BERROCAL, F., GONZÁLEZ-ORTIZ, A. y CENTOLOSI, B. (2017). Evaluación de la eficacia de la formación en la Administración Pública: la transferencia al puesto. Madrid: INAP.

ARTHUR, W., BENNETT, J. B., STANUSH, P. L. y MCNELLY, T. L. (1998). "Factors that influence skill decay and retention: A quantitative review and meta-analysis", Human Performance, 11(1), 57-101. DOI: 10.1207/ s15327043hup1101_3.

AUSTIN, M. J., WEISNER, S., SCHRANDT, E., GLEZOS-BELL, S., y MURTAZA, N. (2006). "Exploring the transfer of learning from an executive development program for human services managers". Administration in Social Work, 30(2), 71-90. DOI: 10.1300\%2FJ147v30n02_06.

AWONIYI, E. A., GRIEGO, O. V. y MORGAN, G. A. (2002). "Person-environment fit and transfer of training", International Journal of Training and Development, 6(1), 25-35. DOI: 10.1111/1468-2419.00147.

AXTELL, C. M., MAITLIS, S. y YEARTA, S. K. (1997). "Predicting immediate and longer-term transfer of training", Personnel Review, 26(3), 201-213. DOI: 10.1108/00483489710161413.

BALDWIN, T. T. y FORD, J. K. (1988). "Transfer of training: A review and directions for future research", Personnel Psychology, 41, 63-105. DOI: 10.1111/j.1744-6570.1988.tb00632.x.

BATES, R. A., KAUFFELD, S. y HOLTON, E. F. (2007). "Examining the factor structure and predictive ability of the german-version of the learning transfer system inventory", Journal of European Industrial Training, 31(3), 195-211. DOI: 10.1108/03090590710739278.

BLUME, B. D., FORD, J. K., BALDWIN, T. T. y HUANG, J. L. (2010). "Transfer of Training: A Meta-Analytic Review", Journal of Management, 36(4), 1065-1105. DOI: 10.1177/0149206309352880.

BRINKERHOFF, R. O. (2006). "Increasing impact of training investments: an evaluation strategy for building organizational learning capability". Industrial and Commercial Training, 38(6), 302-307. DOI: 10.1108/00197850610685824.

BOUD, D. y MIDDLETON, H. (2003). "Learning from others at work: communities of practice and informal learning", Journal of Workplace Learning, 15(5), 194-202. DOI: 10.1108/13665620310483895.

BROAD, M. L. (2005). Beyond transfer of training: Engaging systems to improve performance. San Francisco: John Wiley \& Sons.

BURKE, L. A. y BALDWIN, T. T. (1999). "Workforce training transfer: A study of the effect of relapse prevention training and transfer climate", Human Resource Management, 38(3), 227-241. DOI: 10.1002/(SICl)1099050X(199923)38:3<227::AID-HRM5>3.0.CO;2-M.

BURKE, L. A. y HUTCHINS, H. M. (2008). "A study of best practices in training transfer and proposed model of transfer", Human Resource Development Quarterly, 19(2), 107-128. DOl: 10.1002/hrdq.1230.

CABRERA, F. A. (1993). "La evaluación en las sesiones instructivas". En F. CABRERA, T. DONOSO, y M. A. MARÍN (Eds.), Manual de formación pedagógica básica para formadores (pp. 215-263). Barcelona: PPU.

CHEN, J. y SILVERTHORNE, C. (2008). "The impact of locus of control on job stress, job performance and job satisfaction in Taiwan", Leadership \& Organization Development Journal, 29(7), 572-582. DOI: 10.1108/01437730810906326.

CHENG, E. W. L. y HO, D. C. K. (2001). "A review of transfer of training studies in the past decade", Personnel Review, 30(1), 102-118. DOI: 10.1108/00483480110380163.

CHIABURU, D. S. y LINDSAY, D. R. (2008). "Can do or will do? The importance of self-efficacy and instrumentality for training transfer" Human Resource Development International, 11(2), 199-206. DOI: 10.1080/13678860801933004. 
CHIABURU, D. S. y MARINOVA, S. V. (2005). "What predicts skill transfer? An exploratory study of goal orientation, training self-efficacy and organizational supports", International Journal of Training and Development, 9(2), 110123. DOI: 10.1111/j.1468-2419.2005.00225.x.

CHIABURU, D. S., VAN DAM, K. y HUTCHINS, H. M. (2010). "Social Support in the Workplace and Training Transfer: A longitudinal analysis", International Journal of Selection and Assessment, 18(2), 187-200. DOI: 10.1111/j.14682389.2010.00500.x.

CIRASO, A., ESPONA, B., QUESADA, C., VALDIVIA, P. y PINEDA, P. (2016). "MEEL: Modelo de Evaluación del eLearning en la administración pública", Gestión y Análisis de Políticas Públicas, 15. Recuperado de http:// revistasonline.inap.es/index.php?journal=GAPP\&page=article\&op=view\&path $\% 5 B \% 5 D=10315 \&$ path $\% 5 B \%$ $5 \mathrm{D}=10879$.

CLARKE, N. (2002). "Job/work environment factors influencing training effectiveness within a human service agency: Some indicative support for Baldwin and Fords' transfer climate construct". International Journal of Training and Development, 6(3), 146-162. DOI: 10.1111/1468-2419.00156.

COLQUITT, J. A., LEPINE, J. A. y NOE, R. A. (2000). "Toward an integrative theory of training motivation: A metaanalytic path analysis of 20 years of research". Journal of Applied Psychology, 85(5), 678-707. DOI: 10.1037/00219010.85.5.678.

CROMWELL, S. E. y KOLB, J. A. (2004). "An examination of work-environment support factors affecting transfer of supervisory skills training to the workplace". Human Resource Development Quarterly, 15(4), 449-471. DOI: $10.1002 / \mathrm{hrdq} .1115$.

DEVOS, C., DUMAY, X., BONAMI, M., BATES, R. y HOLTON, E. (2007). "The Learning Transfer System Inventory (LTSI) translated into French: internal structure and predictive validity". International Journal of Training and Development, 11(3), 181-199. DOI: 10.1111/j.1468-2419.2007.00280.x.

FORD, J. K., QUIÑONES, M. A., SEGO, D. J. y SORRA, J. S. (2006). "Factors Affecting The Opportunity To Perform Trained Tasks On The Job”, Personnel Psychology, 45(3), 511-527. DOI: 10.1111/j.1744-6570.1992.tb00858.x.

FOXON, M. (2008). "The Influence of Motivation to Transfer, Action Planning, and Manager Support on the Transfer Process", Performance Improvement Quarterly, 10(2), 42-63. DOI: 10.1111/j.1937-8327.1997.tb00048.x.

FOXON, M. y FOXON, M. (1987). "Transfer of Training - A Practical Application", Journal of European Industrial Training, 11(3), 17-20. DOI: 10.1108/eb002223.

GILPIN-JACKSON, Y. y BUSHE, G. R. (2007). "Leadership development training transfer: a case study of post-training determinants", Journal of Management Development, 26(10), 980-1004. DOI: 10.1108/02621710710833423.

GROSSMAN, R. y SALAS, E. (2011). "The transfer of training: what really matters", International Journal of Training and Development, 15(2), 103-120. DOI: 10.1111/j.1468-2419.2011.00373.x.

HAWLEY, J. D. y BARNARD, J. K. (2005). "Work Environment Characteristics and Implications for Training Transfer: A Case Study of the Nuclear Power Industry", Human Resource Development International, 8(1), 65-80. DOI: 10.1080/1367886042000338308.

HOLLENBECK, G. P. y INGOLS, C. A. (1990). "What's the takeaway?", Training \& Development Journal, 44(7), 83-85.

HOLTON, E. F. (1996). "Final word: Response to reaction to Holton article", Human Resource Development Quarterly, 7(1), 27-29. DOI: 10.1002/hrdq.3920070105.

HOLTON, E. F. (2005). "Holton's evaluation model: New evidence and construct elaborations", Advances in Developing Human Resources, 7(1), 37-54. DOI: 10.1177/1523422304272080.

HOLTON, E. F., BATES, R. A. y RUONA, W. E. A. (2000). "Development of a generalied learning transfer system inventory", Human Resource Development Quartely, núm. 11(4), pag. 333-360. DOI: 10.1002/1532-1096(200024)11:4<333::AIDHRDQ2>3.0.CO;2-P.

HUGHES, A. (2016). "A Meta-Analytic Integration of What Matters in Training Transfer", PhD Thesis, University of Central Florida. Electronic Theses and Dissertations. 4972. http://stars.library.ucf.edu/etd/4972.

INGVARSON, L., MEIERS, M. y BEAVIS, A. (2005). "Factors affecting the impact of professional development programs on teachers' knowledge, practice, student outcomes \& efficacy”, Professional development for teachers and school leaders, 1. DOI: 10.14507/epaa.v13n10.2005.

JUDGE, T. A. y BONO, J. E. (2001). "Relationship of core self-evaluations traits-self-esteem, generalized self-efficacy, locus of control, and emotional stability-with job satisfaction and job performance: A meta-analysis", Journal of Applied Psychology, 86(1), 80-92. DOI: 10.1037/0021-9010.86.1.80.

KIRKPATRICK, D. L. (1959). "Techniques for evaluating training programs", Journal of ASTD, 11, 1-13.

KONTOGHIORGHES, C. (2001). "Factors Affecting Training Effectiveness in the Context of the Introduction of New Technology-A US Case Study", International Journal of Training and Development, 5(4), 248-260. DOI: 10.1111/1468-2419.00137.

KONTOGHIORGHES, C. (2004). "Reconceptualizing the learning transfer conceptual framework: empirical validation of a new systemic model", International Journal of Training and Development, 8(3), 210-221. DOI: 10.1111/j.13603736.2004.00209.x.

KUMPIKAITE, V. (2007). "Human Resource Training Evaluation”. Engineering Economics 5 (55). https://www.ceeol. com/search/article-detail id=293358. 
LAMA, F. J. (2014). "Transferencia de la formación de empleados públicos. Elementos de intervención para el incremento de la transferencia del aprendizaje", Tesis doctoral. Universidad de Sevilla.

LIM, D. H. y JOHNSON, S. D. (2002). "Trainee perceptions of factors that influence learning transfer", International Journal of Training and Development, 6(1), 36-48. DOI: 10.1111/1468-2419.00148.

LONGENECKER, C. O. (2004). "Feature articles maximizing transfer of learning from management education programs", Development and Learning in Organizations: An International Journal, 18(4), 4-6. DOI: $10.1108 / 14777280410544538$.

MACHIN, M. A. y FOGARTY, G. J. (2004). "Assessing the antecedents of transfer intentions in a training context", International Journal of Training and Development, 8(3), 222-236. DOI: 10.1111/j.1360-3736.2004.00210.x.

MARTIN, H. J. (2010). "Workplace climate and peer support as determinants of training transfer", Human Resource Development Quarterly, 21(1), 87-104. DOI: 10.1002/hrdq.20038.

MATHIEU, J. E., TANNENBAUM, S. I. y SALAS, E. (1992). "Influences of individual and situational characteristics on measures of training effectiveness", Academy of Management Journal, 35(4), 828-847. DOI: 10.2307/256317.

NIJMAN, D. J. M., NIJHOF, W. J., WOGNUM, A. A. M. (Ida) y VELDKAMP, B. P. (2006). "Exploring differential effects of supervisor support on transfer of training", Journal of European Industrial Training, 30(7), 529-549. DOI: $10.1108 / 03090590610704394$.

NOE, R. A. (1986). "Trainees' attributes and attitudes: Neglected influences on training effectiveness", Academy of management review, 11(4), 736-749. DOI: 10.5465/AMR.1986.4283922.

PAAS, F. G. W. C. y VAN MERRIËNBOER, J. J. G. (1994). "Variability of worked examples and transfer of geometrical problem-solving skills: A cognitive-load approach", Journal of Educational Psychology, 86(1), $122-133$. DOI: 10.1037/0022-0663.86.1.122.

PEREDA, S., BERROCAL, F. y ALONSO, M. A. (2014). "Bases de Psicología del Trabajo para Gestión de Recursos Humanos". Madrid: Síntesis.

PÉREZ, D. P. D. S. (2008). "La formación como proceso de transferencia al puesto de trabajo de los conocimientos aprendidos: un modelo explicativo aplicado al sector de la restauración”, Tesis doctoral, Universidad de las Palmas de Gran Canaria.

PINEDA, P. (2000). "Evaluación del impacto de la formación en las organizaciones". Educar, (27), 119-133.

PINEDA, P. (2002) (Coord). "Evaluación de la formación en las organizaciones", Gestión de la Formación en las Organizaciones. Barcelona: Ariel.

PINEDA, P., CIRASO, A. y QUESADA, C. (2014). “¿Cómo saber si la formación genera resultados? El modelo FET de evaluación de la transferencia”, Capital Humano, 292, 74-80. https://dialnet.unirioja.es/servlet/ articulo?codigo $=4869068$.

PINEDA, P., QUESADA, C. y CIRASO, A. (2011). "Evaluating Training Effectiveness: results of the FET Model in the Public Administration in Spain", Paper presented at the 7th International Conference on Researching Work and Learning (Shanghai, Dec 4-7, 2011).

PINEDA, P., QUESADA, C. y CIRASO, A. (2013). "Evaluation of Training Transfer Factors: The FET Model", Transfer of Learning in Organizations, 121-144. DOI: 10.1007/978-3-319-02093-8 8.

PINEDA, P., QUESADA, C., ESPONA, B., CIRASO, A. y GARCÍA, N. (2012). "Evaluación de la eficacia de la formación en la administración pública española -ETAPE-: el modelo FET". Recuperado de https://ddd.uab.cat/pub/ estudis/2012/115524/evaefifor_a2012.pdf.

PINEDA, P., QUESADA, C., y MORENO, M. V. (2011). Avaluació de la transferència de la formació a l'Administració Pública de Catalunya, Barcelona: Escola d’Administració Pública de Catalunya. ISBN 978-84-393-8664-3. https:// ddd.uab.cat/record/55238.

QUESADA, C. (2014). “¿Se puede predecir la transferencia de los aprendizajes al lugar de trabajo?: Validación del modelo de predicción de la transferencia", Tesis doctoral. Universidad Autónoma de Barcelona. https://ddd.uab. cat/record/127052.

RENTA-DAVIDS, A. I., JIMÉNEZ-GONZÁLEZ, J. M., FANDOS-GARRIDO, M. y GONZÁLEZ-SOTO, Á. P. (2014). "Transfer of learning: Motivation, training design and learning-conducive work effects", European Journal of Training and Development, 38(8), 728-744. DOI: http://dx.doi.org/10.1108/EJTD-03-2014-0026.

RODRIGUEZ, C. M. (2005). "Qualitative Study of Transfer of Training of Student Employees in a Service Industry", Journal of Hospitality \& Tourism Research, 29(1), 42-66. DOI: 10.1177/1096348004270753.

ROUILLER, J. Z. y GOLDSTEIN, I. L. (1993). "The relationship between organizational transfer climate and positive transfer of training”, Human Resource Development Quarterly, 4(4), 377-390. DOI: 10.1002/hrdq.3920040408.

RUSS-EFT, D. (2002). "A Typology of Training Design and Work Environment Factors Affecting Workplace Learning and Transfer", Human Resource Development Review, 1(1), 45-65. DOI: 10.1177/1534484302011003.

SAKS, A. M. (n.d.). "So what is a good transfer of training estimate? A reply to Fitzpatrick", PsycEXTRA Dataset. DOI: 10.1037/e576922011-004.

SAKS, A. M. y BELCOURT, M. (2006). "An investigation of training activities and transfer of training in organizations", Human Resource Management, 45(4), 629-648. DOI: 10.1002/hrm.20135.

SALAS, E. y STAGL, K. C. (2015). "Design Training Systematically and Follow the Science of Training", Handbook of Principles of Organizational Behavior, 57-84. DOI: 10.1002/9781119206422.ch4. 
SALAS, E., TANNENBAUM, S. I., KRAIGER, K. y SMITH-JENTSCH, K. A. (2012). "The Science of Training and Development in Organizations", Psychological Science in the Public Interest, 13(2), 74-101. DOI: $10.1177 / 1529100612436661$.

SANDUVETE, S. (2008). "Innovaciones metodológicas en la evaluación de la formación continua". Tesis doctoral. Universidad de Sevilla.

SCHINDLER, L. A. y BURKHOLDER, G. J. (2016). "A Mixed Methods Examination of the Influence of Dimensions of Support on Training Transfer", Journal of Mixed Methods Research, 10(3), 292-310. DOI: $10.1177 / 1558689814557132$.

SIASSAKOS, D., FOX, R., CROFTS, J. F., HUNT, L. P., WINTER, C. y DRAYCOTT, T. J. (2011). "The management of a simulated emergency: Better teamwork, better performance”, Resuscitation, 82(2), 203-206. DOI: 10.1016/j. resuscitation.2010.10.029.

THAYER, P. W. y TEACHOUT, M. S. (1995). “A Climate for Transfer Model (No. AL/HR-TP-1995-0035). ARMSTRONG LAB BROOKS AFB TX HUMAN RESOURCES DIRECTORATE.

TZINER, A. y FALBE, C. M. (1993). "Training-Related Variables, Gender and Training Outcomes: A Field Investigation", International Journal of Psychology, 28(2), 203-221. DOI: 10.1080/00207599308247185.

VALENCIA-RODRÍGUEZ, M. (2013). “Generación y transferencia de conocimiento", Ingeniería industrial, 34(2), 178187. Disponible en: <http://scielo.sld.cu/scielo.php?script=sci_arttext\&pid=S1815-59362013000200007\&lng=es\&n rm=iso>. ISSN 1815-5936.

VAN DEN BOSSCHE, P., SEGERS, M. y JANSEN, N. (2010). "Transfer of training: the role of feedback in supportive social networks", International Journal of Training and Development, 14(2), 81-94. DOI: 10.1111/j.14682419.2010.00343.x.

VAN DER KLINK, M., GIELEN, E. y NAUTA, C. (2001). "Supervisory support as a major condition to enhance transfer", International Journal of Training and Development, 5(1), 52-63. DOI: 10.1111/1468-2419.00121.

VELADA, R. y CAETANO, A. (2007). "Training transfer: the mediating role of perception of learning", Journal of European Industrial Training, 31(4), 283-296. DOI: 10.1108/03090590710746441.

VELADA, R., CAETANO, A., MICHEL, J. W., LYONS, B. D. y KAVANAGH, M. J. (2007). "The effects of training design, individual characteristics and work environment on transfer of training", International Journal of Training and Development, 11(4), 282-294. DOI: 10.1111/j.1468-2419.2007.00286.x.

WARR, P. y BUNCE, D. (1995). "Trainee characteristics and the outcomes of open learning", Personnel Psychology, 48(2), 347-375. DOI: 10.1111/J.1744-6570.1995.Tb01761.X. 